\title{
Gut Microbial SNPs Induced by High-Fiber Diet Dominate Nutrition Metabolism and Environmental Adaption of Faecalibacterium prausnitzii in Obese Children
}

\author{
Hui Li', Liping Zhao ${ }^{1,2}$ and Menghui Zhang ${ }^{1 *}$ \\ ${ }^{1}$ State Key Laboratory of Microbial Metabolism and Joint International Research Laboratory of Metabolic and Developmental \\ Sciences, School of Life Sciences and Biotechnology, Shanghai Jiao Tong University, Shanghai, China, ${ }^{2}$ Ministry \\ of Education Key Laboratory for Systems Biomedicine, Shanghai Centre for Systems Biomedicine, Shanghai Jiao Tong \\ University, Shanghai, China
}

OPEN ACCESS

Edited by:

Teresa Nogueira, Instituto Nacional Investigaciao Agraria e Veterinaria (INIAV), Portugal

Reviewed by:

Farzam Vaziri,

Pasteur Institute of Iran (PII), Iran

Sandrine Auger,

Le nouvel Institut nationa

de recherche sur l'agriculture, l'alimentation et l'environnement en France INRAE, France

*Correspondence: Menghui Zhang mhzhang@sjtu.edu.cn

Specialty section: This article was submitted to Systems Microbiology, a section of the journa Frontiers in Microbiology

Received: 22 March 2021 Accepted: 23 April 2021 Published: 31 May 2021

Citation: Li H, Zhao L and Zhang M (2021) Gut Microbial SNPs Induced by High-Fiber Diet Dominate Nutrition Metabolism and Environmental Adaption of Faecalibacterium prausnitzii in Obese Children. Front. Microbiol. 12:683714. doi: 10.3389/fmicb.2021.683714
Dietary intervention is effective in human health promotion through modulation of gut microbiota. Diet can cause single-nucleotide polymorphisms (SNPs) to occur in the gut microbiota, and some of these variations may lead to functional changes in human health. In this study, we performed a systematic SNP analysis based on metagenomic data collected from children with Prader-Willi syndrome (PWS, $n=17$ ) and simple obese (SO) children $(n=19)$, who had better healthy conditions after receiving high-fiber diet intervention. We found that the intervention increased the SNP proportions of Faecalibacterium, Bifidobacterium, and Clostridium and decreased those of Bacteroides in all children. Besides, the PWS children had Collinsella increased and Ruminococcus decreased, whereas the SO had Blautia and Escherichia decreased. There were much more BiasSNPs in PWS than in SO (4,465 vs 303), and only 81 of them appeared in both groups, of which 78 were from Faecalibacterium prausnitzii, and 51 were nonsynonymous mutations. These nonsynonymous variations were mainly related to pathways of environmental adaptation and nutrition metabolism, particularly to carbohydrate and nucleotide metabolism. In addition, dominant strains carrying BiasSNPs in all children shifted from F. prausnitzii AF32-8AC and F. prausnitzil 942/30-2 to F. prausnitzii SSTS Bg7063 and F. prausnitzii JG BgPS064 after the dietary intervention. Furthermore, although the abundance of Bifidobacterium increased significantly by the intervention and became dominant strains responsible for nutrition metabolism, they had less BiasSNPs between the pre- and post-intervention group in comparison with Faecalibacterium. The finding of $F$. prausnitzii as important functional strains influenced by the intervention highlights the superiority of applying SNP analysis in studies of gut microbiota. This study provided evidence and support for the effect of dietary intervention on gut microbial SNPs, and gave some enlightenments for disease treatment.

Keywords: SNP, gut microbiota, high-fiber diet, non-synonymous, obese children, metagenome 


\section{INTRODUCTION}

Single-nucleotide polymorphism (SNP) is the most common genetic variation in DNA sequence in order to better adapt to the external environment in the evolutionary process (Haraksingh and Snyder, 2013). SNPs in the coding region can be classified as nonsynonymous mutation and synonymous mutation. Nonsynonymous mutation changes the sequence of amino acids and then affects the genetic function, while the synonymous mutation does not affect the genetic function (Tennessen, 2008). There are huge amounts of microorganisms living in the human intestinal tract, and the diet is one of the most important factors shaping the structure and function of the gut microbiota (Goldsmith and Sartor, 2014; Shen, 2017). Environmental pressure caused by the change in the diet not only alter the structure of the gut microbiota but also led to genetic variations in the microbes (Truong et al., 2017). These variations can lead to different functions in strains, which, in turn, affect the health of the host.

Considering the taxonomic diversity of bacteria and the genetic variations in response to constant environmental change (Hofreiter et al., 2015), the analyses based on the abundance and composition of the gut microbiota are not enough to reflect changes in gene function or microbial transfer, which might not only omit some correlations but also infer wrong conclusions from this rough quantitative level. For instance, researchers had studied the gut metagenomes of 98 mothers and their infants over 1 year, used rare SNPs to reveal vertical transmission of strains, and found that the colonization with strains of infants mainly derived from the environment but not from their mothers, although the gut microbial composition of infants converged toward that of their mothers over time (Nayfach et al., 2016). This pattern might be missed in the analysis with the gut microbial composition, and it was mistakenly assumed that vertical bacterial transmission of infants from their mother was increased during the first year after birth.

Single-nucleotide polymorphisms, which refer to singlenucleotide variations in genes, are more able to reveal the differences between strains and between genes. The continuous expansion of gut metagenomic sample dataset and an increasing number of the bacterial reference genome have facilitated the studies of gut microbial SNPs. The flexible application of gut microbial SNPs can solve the complex problems that other analysis at species level cannot solve, obtain more accurate results at the strain- or gene-level, and provide new clues for the precision diagnosis and treatment of diseases (GallowayPeña et al., 2012; Leonard et al., 2016; Zou et al., 2020). Patient-specific SNPs were found in the gut microbiota of both type 2 diabetes mellitus (T2D) and tuberculosis patients, which could separate the patients from the healthy individuals. The gene carrying T2D-specific SNPs encodes the alpha glucoside enzyme, which is a kind of important T2D drug target, and the researchers believed that these SNPs could be used as drug targets for the treatment of T2D (Chen et al., 2017). The tuberculosis-specific SNP genes were mainly involved in carbohydrate metabolism prevalently from Bacteroides vulgatus, suggesting that there were altered carbohydrate preference and different carbohydrate metabolism patterns in the gut of tuberculosis patients, and providing reference for the diagnosis and treatment of tuberculosis (Hu et al., 2019). Other researchers conducted gut microbial SNP studies on antibiotic resistance genes of individuals from different countries and found that the population-specific SNPs on antibiotic resistance genes were not related to the country, but might be attributed to the altered microbiota by differences in population structure or different antibiotic usage (Hu et al., 2013).

Many studies have proved the close relationship between the gut microbiota and obesity, but in-depth researches on the strain- or gene-level still need to be conducted (Turnbaugh et al., 2006; Flint, 2011; Baothman et al., 2016). Our previous study demonstrated that a high-fiber dietary intervention significantly improved the physiological conditions of the genetic (PraderWilli syndrome, PWS) and simple obese (SO) children, and this promotion was found to be relevant to the change in the gut microbiota (Zhang et al., 2015). In order to better understand the underlying mechanism for this effective treatment, we performed a systemic SNP analysis based on high-throughput metagenomic sequencing data obtained from two longitudinal cohorts, children with PWS or SO. We first identified SNPs in each cohort affected by the intervention and screened out genes with significant change in SNP density. The species that carried these genes were then sourced and linked with relevant metabolic pathways. After that, we focused on BiasSNPs that occurred in both cohorts, particularly on those nonsynonymous mutations. Functional pathways and dominant strains with BiasSNPs influenced by the intervention were investigated further. Finally, PWS and SO-specific BiasSNP-affected strains and metabolic pathways were individually analyzed.

\section{MATERIALS AND METHODS}

\section{Data Collection}

The dietary intervention trial was approved by the Ethics Committee of the School of Life Sciences and Biotechnology, Shanghai Jiao Tong University, with No. 2012-016 and registered at the Chinese Clinical Trial Registry with No. ChiCTR-ONC12002646. Written informed consent was obtained from the guardian of the obese children. The trial was performed as described in the previous study (Zhang et al., 2015). Briefly, 17 PWS and 19 SO children completed the dietary intervention in the hospital for 90 and 30 days, respectively. The diet used in the clinical trial for intervention mainly included whole grains, traditional Chinese medicinal foods, and prebiotics (WTP), incorporated certain amount of vegetables, fruits, and nuts (Xiao et al., 2014).

The fecal samples and physiological indexes of all obese children were collected at predefined time points (PWS: on intervention days $0,30,60$, and 90; SO: days 0 and 30) (Zhang et al., 2015). Metagenomic sequencing of the extracted and purified DNA was performed on Illumina Hiseq 2000 platform at Shanghai Biotechnology Co., Ltd. All potential biologically hazardous materials in this study were properly handled according to Chinese biosafety laws and regulations. 
The raw metagenome sequencing data was accessed at the NCBI SRA (Sequence Read Archive) database with accession number SRP045211 (Zhang et al., 2015).

\section{Detection of Single-Nucleotide Polymorphisms}

The data pre-processing was done as described in our previous study (Zhang et al., 2015). Briefly, the original sequencing data was quality controlled by FlexBar and Prinseq (Schmieder and Edwards, 2011; Dodt et al., 2012), and then aligned to the human genome reference (Homo sapiens, UCSC hg19) using Bowtie2 to remove the reads from human (Langmead and Salzberg, 2012). Each sample had $84.6 \pm 21.2$ million (mean \pm SD) high-quality reads on average. Then BWA (Li and Durbin, 2009) was used to align the high-quality reads to the integrated gene catalog (IGC), which contained approximately 11 million high-quality human gut microbial reference genes (Li et al., 2014; Xie et al., 2016). Afterward, the SAMTools was used to detect, filter, sort, and merge SNPs (Li et al., 2009; Li, 2011).

To ensure the reliability of the detected SNPs, only SNPs with at least five supported sequencing reads were kept, and those with less than $60 \%$ coverage in a group were furtherly removed to achieve more representative SNPs. The downstream analyses were all performed with the SNPs resulting from this procedure.

\section{Calculation of Single-Nucleotide Polymorphisms Density}

Single-nucleotide polymorphism density reflected the number of SNPs per kilobase in a gene per million sequencing pairedend reads. For any sample $S$, the SNP density $D_{i}$ of gene $i$ was calculated as follows:

$$
D_{i}=\frac{n_{i}}{T_{S} L_{i}}
$$

where $n_{i}$ is the number of SNPs in gene $i, T_{s}$ is the sequencing amount of sample $S$, and $L_{i}$ is the length of the gene $i$ per kilobase in sequence.

\section{Detection of BiasSNP}

Detection of BiasSNP was performed through comparison of an SNP in a nucleotide base between two groups. A BiasSNP was the differential SNP that dominated between two groups (Supplementary Figure 1). Nonsynonymous BiasSNP, whose mutation in a gene caused the encoded amino acid to be changed, was furtherly identified by in-house Perl script.

\section{Statistical Analysis}

In this study, the significance of the difference was judged with Wilcoxon paired test for a cohort between before and after the dietary intervention or with Wilcoxon unpaired test for two cohorts in $\mathrm{R}$ software (version 3.5.3). The significance of the difference among multiple groups was tested with permutational multivariate analysis of variance (perMANOVA) in the "vegan" library of the $\mathrm{R}$ software. The phylogenetic and clustering trees based on BiasSNPs were constructed using the maximum likelihood model GTRGAMMA and 1,000 bootstrap replicates in RAxML (Stamatakis, 2014). Additionally, the $\mathrm{R}$ package
"clusterProfiler" (version 3.8.1) was used to perform enrichment analysis of the SNP genes (Yu et al., 2012).

\section{Data Visualization}

Data visualization was mainly realized in the $\mathrm{R}$ software, "ggalluvial" (version 0.11.3), which was used to illustrate the alluvial diagram between strain and metabolic pathway, while dotplot, boxplot, and pieplot were displayed by means of "ggplot2" (version 3.2.0). In addition, the network charts were drawn using the Cytoscape software (version 3.7.2) (Shannon et al., 2003), and the optimization of the tree diagram was accomplished with the aid of the online tool EvolView ${ }^{1}$.

\section{RESULTS}

\section{The Overall Effect of High-Fiber Dietary Intervention on Gut Microbial Single-Nucleotide Polymorphisms in Obese Children}

A total of 218,343 SNPs were detected in the gut microbiota of the 36 obese children. These SNPs were concentrated in 40,515 genes, and these genes could be sourced from 57 genera and 150 species. The high-fiber dietary intervention lessened the overall SNP numbers on the 30th intervention day; the SNPs in PWS children decreased to 85,769 from 109,139 , while in SO children, they decreased to 36,247 from 80,773 . Before the intervention, all children had the dominant SNPs at genus level, which were from Faecalibacterium, Ruminococcus, and Bacteroides. In addition, the SO children had SNPs from Escherichia with relatively higher proportion (33.76\%). The intervention increased the SNP proportions of Faecalibacterium, Bifidobacterium, and Clostridium and decreased those of Bacteroides in all children. Meanwhile, the PWS children had Collinsella increased and Ruminococcus decreased, whereas the SO had Blautia and Escherichia decreased (Figure 1A).

\section{The Altered Single-Nucleotide Polymorphism Density Structure in Prader-Willi Syndrome and Simple Obese Children}

In our previous study, the PWS group had worse health conditions such as higher inflammation level than the SO group before the intervention. However, we did not detect significant difference between the two groups in gut microbial structure (Zhang et al., 2015). Interestingly, with the SNP density structure, a significant separation between the two groups before the intervention was observed (Figure 1B, PerMANOVA test, $P<0.001$ ). The SNP density structure was altered significantly by the dietary intervention in both groups (Figure 1B, PerMANOVA test, $P<0.001$ ). According to the changes that occurred in the PWS group, the structure alteration might occur mainly in the earlier stage of the intervention, as the shift on the 60th and 90th days was less that on the 30th day.

\footnotetext{
${ }^{1}$ https://www.evolgenius.info/evolview
} 


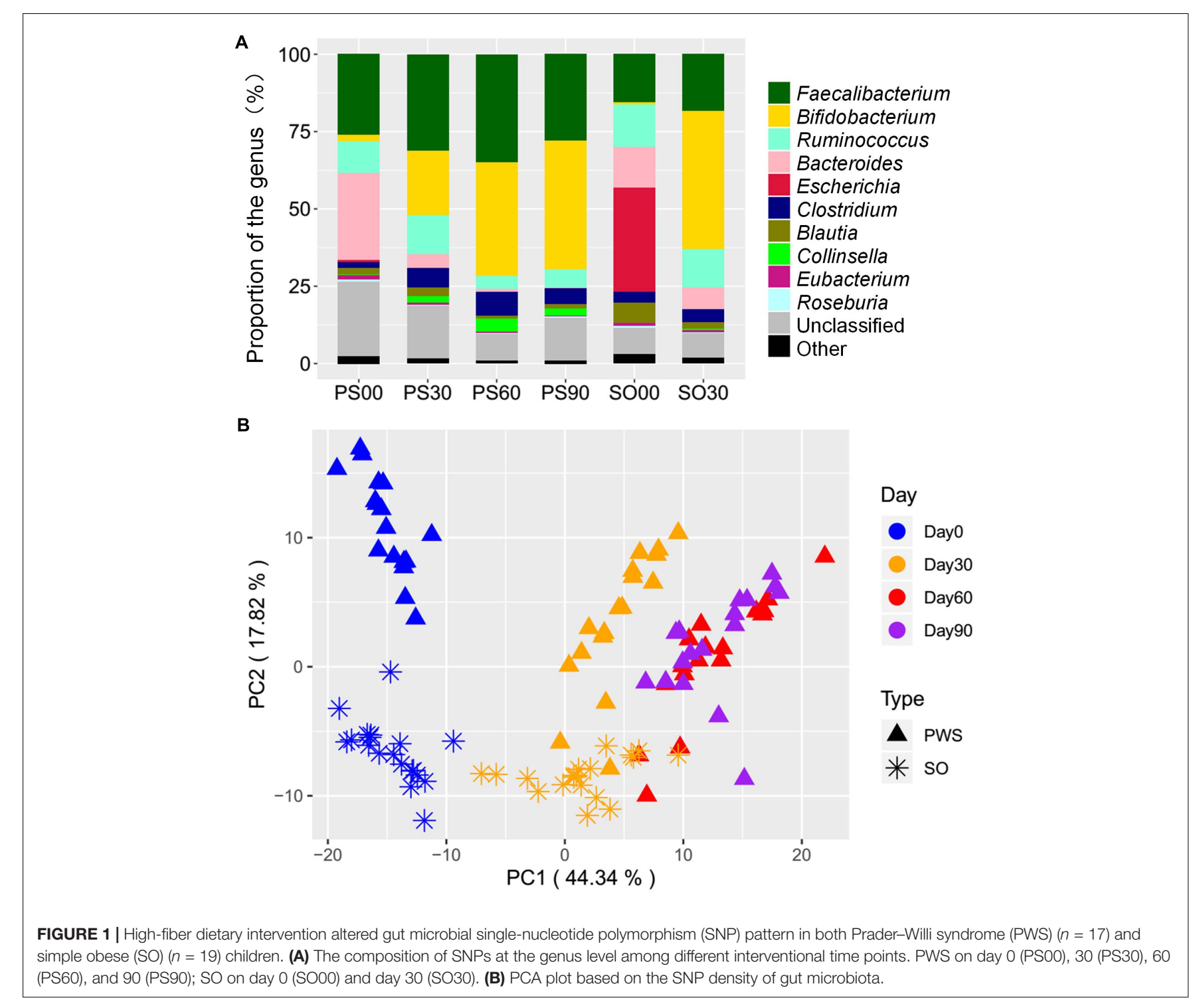

After 30 days of dietary intervention, the PWS children had 26,174 genes significantly changed in SNP density (Wilcoxon test, adjusted $P<0.05)$, and most of them $(20,279)$ had fold changes lager than 8. Among these genes, 13,450 had higher SNP density before the intervention, which were distributed in 102 species and mainly concentrated in Faecalibacterium prausnitzii (20.27\%), Bacteroides stercoris (15.63\%), and Bacteroides dorei (12.49\%). These genes were enriched in metabolic pathways for amino acid biosynthetic (ko01230), carbon metabolism (ko01200), two-component system (ko02020), and starch and sucrose metabolism (ko00500) (Figure 2A). After the intervention, 6,847 genes had higher SNP density. These genes were concentrated in 59 strains, mainly including butyrate-producing bacterium (24.65\%), Bifidobacterium catenulatum (17.95\%), and Bifidobacterium longum (10.60\%). The corresponding metabolic pathways contained biosynthesis of amino acids (ko01230), carbon metabolism (ko01200), and starch and sucrose metabolism (ko00500) (Figure 2B). Notably, although the enriched metabolic pathways remain constant, their contributing strains changed after the intervention. For instance, the biosynthesis of amino acids (ko01230) was mainly from F. prausnitzii before the intervention, while the contributing strains of this function were replaced by butyrate-producing bacterium, $B$. catenulatum, and B. longum after the intervention.

The SO children had 17,427 genes significantly changed in SNP density (adjusted $P<0.05$ ). The number of genes with fold change greater than 8 was 13,927 . Among them, 10,409 genes with higher SNP density existed in the preintervention group, which were derived from 112 strains and mainly in Escherichia coli (37.53\%), F. prausnitzii (16.88\%), and Ruminococcus sp. 5_1_39BFAA (11.84\%). Though the distribution of these strains in SO were different from that in PWS, these SNP density differential genes they carried also focused on biosynthesis of amino acids (ko01230), carbon metabolism (ko01200), and twocomponent system (ko02020) (Figure 2C), while 3,518 
A
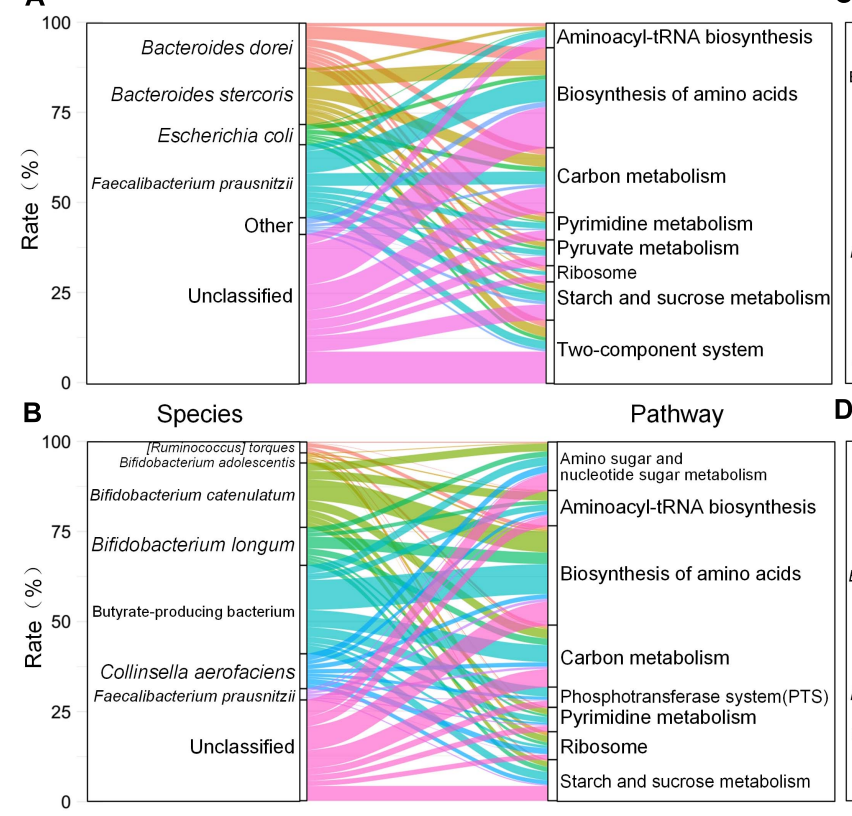

c

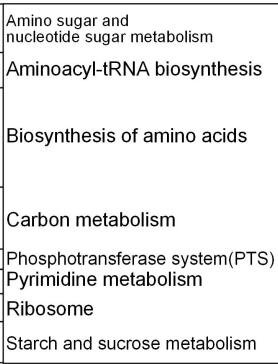

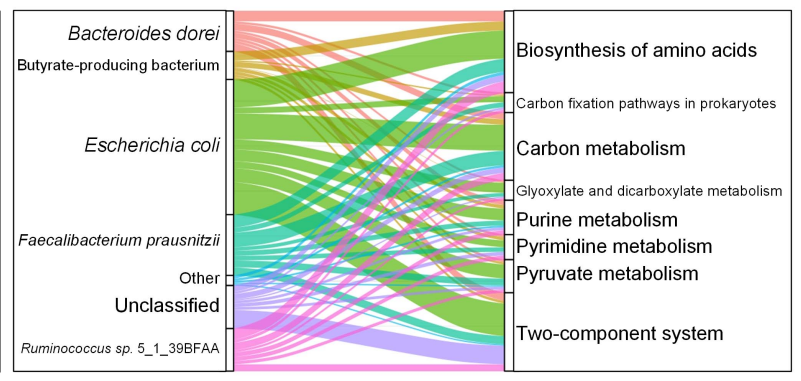

D Species

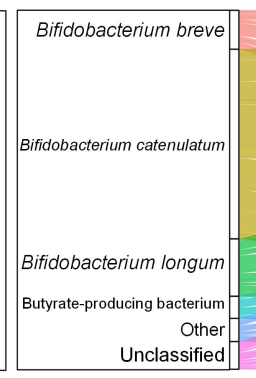

Pathway

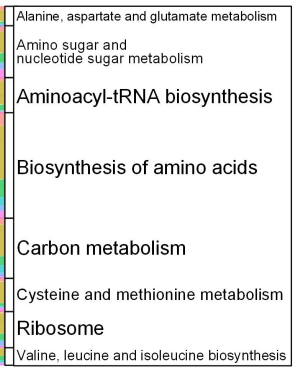

FIGURE 2 | Species and metabolic pathways corresponding to the genes with differential SNP density between before and after intervention in obese children. (A) The corresponding species and metabolic pathways of the genes with high SNP density in PWS before the intervention. (B) The corresponding species and metabolic pathways of the genes with high SNP density in PWS after the intervention. (C) The corresponding species and metabolic pathways of the genes with high SNP density in SO before the intervention. (D) The corresponding species and metabolic pathways of the genes with high SNP density in SO after the intervention.

genes with higher SNP density were detected in the postintervention group, which were derived from 26 strains and mainly in B. catenulatum (52.84\%), B. longum (15.98\%), and Bifidobacterium breve (10.83\%). These genes focused on biosynthesis of amino acids (ko01230), carbon metabolism (ko01200), and amino sugar and nucleoside sugar metabolism (ko00520) (Figure 2D). Similar to the PWS, the intervention also changed the relationships between the strains and the metabolic pathways. However, unlike in PWS, E. coli followed by $F$. prausnitzii were the main contributors in $\mathrm{SO}$ to the biosynthesis of amino acids before the intervention, while contributions from these two strains might be neglected, and $B$. catenulatum took dominant responsibilities after the dietary intervention.

\section{Common BiasSNPs Before and After Intervention in Prader-Willi Syndrome and Simple Obese Children}

With the interest in the differences of SNP between the groups in sequence, we furtherly screened BiasSNPs whose variation were dominant in one group/one time point among more than $60 \%$ of the individuals. Comparing with the SNPs before and after 30 days of intervention, the detected BiasSNPs in PWS was 4,465, larger than 303 in SO. The PWS and SO had only 81 BiasSNPs in common distributed in 69 genes. Source track indicated that 78 common BiasSNPs were from F. prausnitzii, and the remaining three were from Streptococcus thermophilus, suggesting that F. prausnitzii was the most affected under the intervention.
In order to identify the source of these BiasSNPs at genome level, 103 genomes of $F$. prausnitzii were downloaded from the GenBank database, and the nucleotide sites corresponding to common BiasSNPs were abstracted. Only nine out of the 103 F. prausnitzii strains had more than $70 \%$ coverage of BiasSNPs. Then, we constructed a phylogenetic tree of these nine F. prausnitzii and the corresponding strains from the preand post-intervention groups based on these 78 BiasSNPs. It was observed that $F$. prausnitzii AF32-8AC was closest to the pre-intervention group, followed by $F$. prausnitzii 942/30-2, F. prausnitzii APC942/18-1, and F. prausnitzii MGYG-HGUT02545 in the phylogenetic tree (Figure 3A), while the closest to the post-intervention group was F. prausnitzii SSTS Bg7063, followed by $F$. prausnitzii JG BgPS064 and F. prausnitzii NZ FPSSTS7063 SV a2 mod. This suggested that the dominant strains of $F$. prausnitzii were converted from $F$. prausnitzii AF32-8AC and F. prausnitzii 942/30-2 to F. prausnitzii SSTS Bg7063 and F. prausnitzii JG BgPS064 after the dietary intervention.

Of the 81 common BiasSNPs, 53 were nonsynonymous mutations in which 51 were in $F$. prausnitzii and the remaining two were in S. thermophilus. These 53 nonsynonymous BiasSNPs existed in 49 genes, whose detailed information are listed in Table 1. The enriched KEGG metabolic pathways based on these 49 genes showed that these SNPs were mainly related to nutrition metabolism and environmental adaptation functions (Figures 3B,C). In detail, pathways related to nutrition metabolism included carbohydrate metabolism (ko00720), nucleotide metabolism (ko09104), amino acid metabolism (ko09105), lipid metabolism (ko09103), 
A

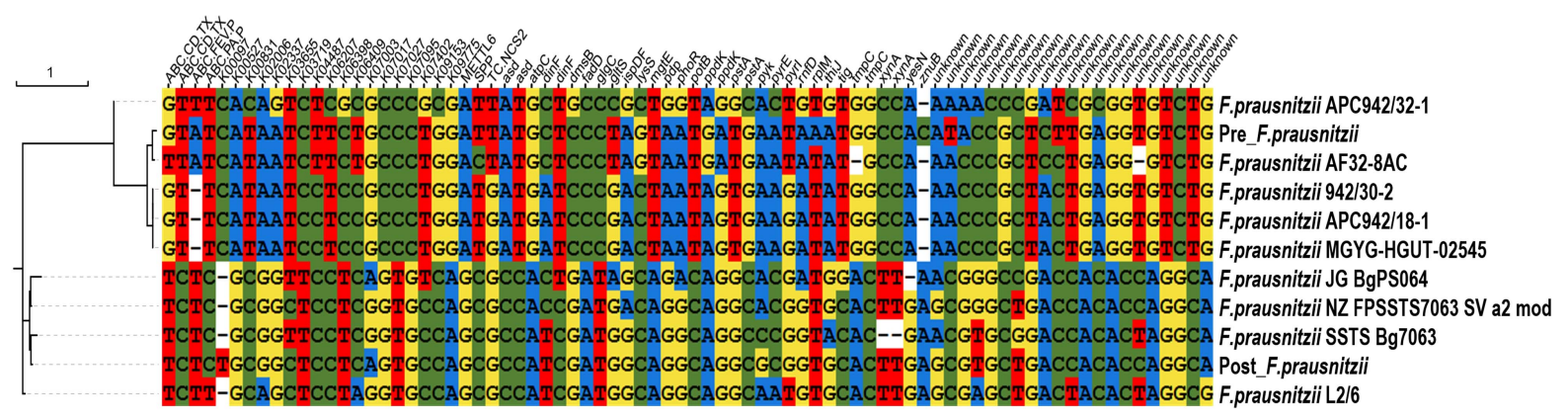

B

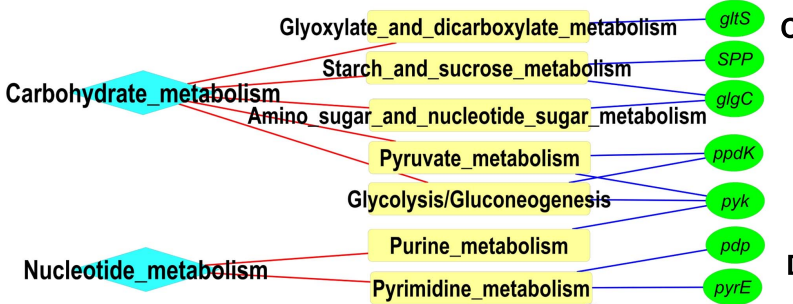

Alanine,aspartate_and_glutamate_metabollsm pyrl

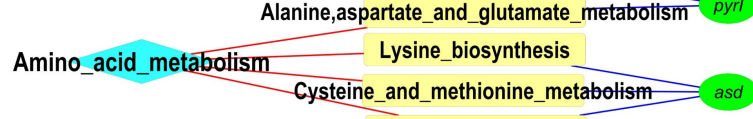

Glycine,serine_and_threonine_metabolism

Lipid_metabolism

Fatty_acid_biosynthesis

Fatty_acid_degradation

Terpenoid_backbone_biosynthesis

Metabolism_of_terpenoids and_polyketides
C

D

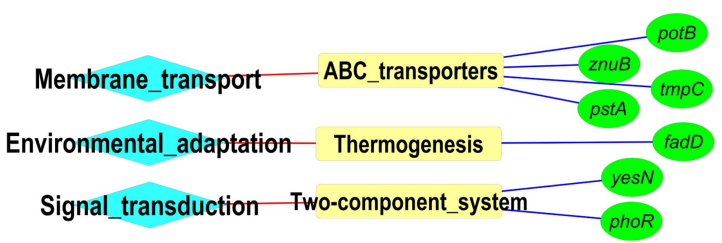

E

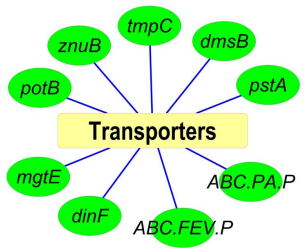

FIGURE 3 | Strains and metabolic pathways corresponding to common BiasSNPs. (A) Phylogenetic relationship of nine Faecalibacterium prausnitzii and the corresponding strains of pre- and post-intervention groups based on the common BiasSNPs. (B) Metabolic pathways related to nutrition metabolism. (C) Metabolic pathways associated with environmental adaptation. (D) Metabolic pathways associated with the gene fadD. (E) Genes encoding transporters.

and metabolism of terpenoids and polyketides (ko09109). Particularly, there were more genes related to carbohydrate and nucleotide metabolism (Figure 3B). Pathways associated with environmental information processing included membrane transport (ko09131), environmental adaptation (ko09159), and signal transduction (ko09132), which were mainly linked with ABC transporters (ko02010), thermogenesis (ko04714), and two-component system (ko02020), respectively (Figure 3C).

Notably, some genes, such as $p y k$ and $f a d D$, were involved in multiple metabolic pathways (Figures 3B,D), suggesting their important roles in the entire metabolic network. The gene $p y k$ encodes pyruvate kinase (Gubler et al., 1994), mainly taking part in glycolysis/gluconeogenesis (ko00010), purine metabolism (ko00230), and pyruvate metabolism (ko00620). The gene fadD encodes long-chain acyl-CoA synthetase that can use long-chain fatty acids as carbon source and energy (Pech-Canul et al., 2020), and is mainly involved in fatty acid biosynthesis and degradation (ko00061 and ko00071), ferroptosis (ko04216), lipid biosynthesis proteins (ko01004), quorum sensing (ko02024), thermogenesis (ko04714), etc.

There were several gene-encoding transporters in enrichment pathways (Figure 3E). These transporters include a variety of proteins that are involved in signal transduction and various intracellular processes, such as cell proliferation and differentiation. In this study, the genes with common BiasSNPs encode a variety of transport system permease proteins, such as spermidine/putrescine transport system permease protein ( $p o t B$ encoding), phosphate transport system permease protein ( $p s t A$ encoding), polar amino acid transport system permease protein (ABC.PA.P encoding), iron complex transport system permease protein (ABC.FEV.P encoding), zinc transport system permease protein ( $z n u B$ encoding), etc. Besides, they also encode basic membrane protein A ( $b m p A$ encoding), multidrug resistance protein ( $\operatorname{din} F$ encoding), and anaerobic dimethyl sulfoxide reductase subunit $\mathrm{B}$ ( $d m s B$ encoding). These results indicated that these genes with nonsynonymous SNPs were closely related to the transporters under the dietary intervention.

\section{Prader-Willi Syndrome-Specific BiasSNPs Affected by High-Fiber Dietary Intervention}

PWS-specific BiasSNPs $(4,384)$ were detected between the preand post-intervention groups, which were distributed in 2,039 genes and sourced from 34 strains. The distribution at strain level indicated that most of the BiasSNPs were derived from F. prausnitzii (82.0\%) (Figure 4A). 
TABLE 1 | Information of 49 genes with nonsynonymous BiasSNP.

\begin{tabular}{|c|c|c|}
\hline GenelD & Name & Definition \\
\hline $\begin{array}{l}\text { SZEY- } \\
\text { 27A_GL0066464 }\end{array}$ & $A B C . C D . T X$ & HlyD family secretion protein \\
\hline MH0423_GL0087716 & ABC.FEV.P & $\begin{array}{l}\text { Iron complex transport system } \\
\text { permease protein }\end{array}$ \\
\hline MH0204_GL0062877 & ABC.PA.P & $\begin{array}{l}\text { Polar amino acid transport system } \\
\text { permease protein }\end{array}$ \\
\hline $\begin{array}{l}\text { O2.UC34- } \\
\text { 2_GL0007607 }\end{array}$ & $A C S L, f a d D$ & Long-chain acyl-CoA synthetase \\
\hline MH0094_GL0105570 & asd & $\begin{array}{l}\text { Aspartate-semialdehyde } \\
\text { dehydrogenase }\end{array}$ \\
\hline $\begin{array}{l}\text { SZEY- } \\
\text { 58A_GL0041727 }\end{array}$ & $\begin{array}{l}\text { ATPF1E, } \\
\operatorname{atpC}\end{array}$ & $\begin{array}{l}\text { F-type } \mathrm{H}+\text {-transporting ATPase } \\
\text { subunit epsilon }\end{array}$ \\
\hline NOM017_GL0035853 & $\begin{array}{l}b m p A \\
b m p B \\
t m p C\end{array}$ & $\begin{array}{l}\text { Basic membrane protein } A \text { and related } \\
\text { proteins }\end{array}$ \\
\hline $\begin{array}{l}\text { SZEY- } \\
\text { 106A_GL0033468 }\end{array}$ & $d m s B$ & $\begin{array}{l}\text { Anaerobic dimethyl sulfoxide } \\
\text { reductase subunit B }\end{array}$ \\
\hline MH0161_GL0016845 & $\begin{array}{l}\text { E3.2.1.8, } \\
\text { xynA }\end{array}$ & Endo-1,4-beta-xylanase \\
\hline MH0161_GL0083263 & $g \operatorname{lgC}$ & $\begin{array}{l}\text { Glucose-1-phosphate } \\
\text { adenylyltransferase }\end{array}$ \\
\hline $\begin{array}{l}\text { T2D- } \\
\text { 109A_GL0053344 }\end{array}$ & GLU, gltS & Glutamate synthase (ferredoxin) \\
\hline $\begin{array}{l}\text { 250twins_37179_ } \\
\text { GL0047337 }\end{array}$ & ispDF & $\begin{array}{l}\text { 2-C-methyl-D-erythritol 4-phosphate } \\
\text { Cytidylyltransferase/2-C-methyl-D- } \\
\text { erythritol 2,4-Cyclodiphosphate } \\
\text { synthase }\end{array}$ \\
\hline BGI-28A_GL0080202 & K07027 & Glycosyltransferase 2 family protein \\
\hline MH0260_GL0085944 & K09153 & Small membrane protein \\
\hline MH0427_GL0005657 & KARS, lysS & Lysyl-tRNA synthetase, class II \\
\hline V1.Fl16_GL0163211 & METTL6 & Methyltransferase-like protein 6 \\
\hline MH0089_GL0042766 & mgtE & Magnesium transporter \\
\hline NLM015_GL0035022 & $p d p$ & Pyrimidine-nucleoside phosphorylase \\
\hline MH0222_GL0152632 & phoR & $\begin{array}{l}\text { Two-component system, OmpR family, } \\
\text { phosphate regulon sensor histidine } \\
\text { kinase PhoR }\end{array}$ \\
\hline BGI-06A_GL0076090 & $P K$, pyk & Pyruvate kinase \\
\hline T2D-59A_GL0116703 & potB & $\begin{array}{l}\text { Spermidine/putrescine transport } \\
\text { system permease protein }\end{array}$ \\
\hline MH0251_GL0137853 & ppdK & Pyruvate, orthophosphate dikinase \\
\hline MH0055_GL0043341 & pstA & $\begin{array}{l}\text { Phosphate transport system } \\
\text { permease protein }\end{array}$ \\
\hline MH0069_GL0033002 & pyrE & Orotate phosphoribosyltransferase \\
\hline T2D-56A_GL0037409 & pyrl & $\begin{array}{l}\text { Aspartate carbamoyltransferase } \\
\text { regulatory subunit }\end{array}$ \\
\hline $\begin{array}{l}\text { 250twins_36674_ } \\
\text { GL0060378 }\end{array}$ & $r n f D$ & $\begin{array}{l}\mathrm{Na}+\text {-translocating ferredoxin:NAD+ } \\
\text { oxidoreductase subunit } \mathrm{D}\end{array}$ \\
\hline $\begin{array}{l}\text { SZEY- } \\
\text { 103A_GL0004639 }\end{array}$ & $\begin{array}{l}\text { RP-L13, } \\
\text { MRPL13, } \\
\text { rpIM }\end{array}$ & Large subunit ribosomal protein L13 \\
\hline $\begin{array}{l}\text { V1.CD6-0- } \\
\text { PT_GL0047319 }\end{array}$ & SPP & Sucrose-6-phosphatase \\
\hline $\begin{array}{l}\text { SZEY- } \\
\text { 90A_GL0013477 }\end{array}$ & $\begin{array}{l}\text { TC.MATE, } \\
\text { SLC47A, } \\
\text { norM }\end{array}$ & $\begin{array}{l}\text { Multidrug resistance protein, MATE } \\
\text { family }\end{array}$ \\
\hline MH0176_GL0049322 & TC.NCS2 & $\begin{array}{l}\text { Nucleobase:cation symporter-2, NCS2 } \\
\text { family }\end{array}$ \\
\hline MH0422_GL0084041 & this & Protein deglycase \\
\hline $\begin{array}{l}\text { 160400887- } \\
\text { stool1_196973 }\end{array}$ & tig & Trigger factor \\
\hline
\end{tabular}

(Continued)
TABLE 1 | Continued

\begin{tabular}{|c|c|c|}
\hline GenelD & Name & Definition \\
\hline T2D-10A_GL0004234 & yesN & $\begin{array}{l}\text { Two-component system, response } \\
\text { regulator YesN }\end{array}$ \\
\hline $\begin{array}{l}\text { 763678604- } \\
\text { stool1_204596 }\end{array}$ & znuB & $\begin{array}{l}\text { Zinc transport system permease } \\
\text { protein }\end{array}$ \\
\hline MH0136_GL0032411 & K07003 & Uncharacterized protein \\
\hline N084A_GL0010742 & K07017 & Uncharacterized protein \\
\hline $\begin{array}{l}\text { V1.UC35- } \\
\text { 4_GL0167766 }\end{array}$ & K07095 & Uncharacterized protein \\
\hline $\begin{array}{l}\text { T2D- } \\
\text { 198A_GL0043098 }\end{array}$ & K09775 & Uncharacterized protein \\
\hline V1.FI20_GL0181809 & Unclassified & Unclassified \\
\hline DOM026_GL0058508 & Unclassified & Unclassified \\
\hline MH0136_GL0100087 & Unclassified & Unclassified \\
\hline $\begin{array}{l}\text { O2.UC34- } \\
\text { 2_GL0069427 }\end{array}$ & Unclassified & Unclassified \\
\hline $\begin{array}{l}\text { V1.UC27- } \\
\text { O_GL0047860 }\end{array}$ & Unclassified & Unclassified \\
\hline $\begin{array}{l}\text { V1.CD2-0- } \\
\text { PN_GL0116497 }\end{array}$ & Unclassified & Unclassified \\
\hline N051A_GL0048400 & Unclassified & Unclassified \\
\hline 264199.stu_r17 & Unclassified & Unclassified \\
\hline MH0094_GL0121652 & Unclassified & Unclassified \\
\hline NLF010_GL0004489 & Unclassified & Unclassified \\
\hline MH0184_GL0028587 & Unclassified & Unclassified \\
\hline
\end{tabular}

A network of the KEGG metabolic pathways, with the nonsynonymous BiasSNPs uniquely occurring in the PWS children, was constructed. This network showed that these BiasSNPs were mainly relevant to nutrition metabolism and environmental adaptation (Figure 4B). There were more BiasSNPs in PWS than in SO, and the metabolic functions of these PWS-specific BiasSNPs were similar to that of the common BiasSNPs, indicating that the gut microbial SNPs were more susceptible by dietary intervention in PWS. Nutrition metabolism mainly included carbohydrate metabolism, amino acid metabolism, metabolism of cofactors and vitamins, nucleotide metabolism, and lipid metabolism. Pathways of environmental adaptation mainly covered cellular community, membrane transport, and folding, sorting, and degradation. Genes (242) with nonsynonymous BiasSNPs in the pathway network of PWS children are listed in Supplementary Table 1.

\section{Simple Obese-Specific BiasSNPs Affected by High-Fiber Dietary Intervention}

There were 222 SO-specific BiasSNPs between before and after dietary intervention, far fewer than those in PWS. These BiasSNPs were distributed in 196 genes and sourced from 17 strains, also mainly from $F$. prausnitzii (74.8\%) (Figure 5A). Among them, 104 BiasSNPs were nonsynonymous. The network of enriched KEGG metabolic pathways constructed with the genes carrying nonsynonymous BiasSNPs showed that these BiasSNPs were mainly related to nutrition metabolism, DNA replication and repair, and translation (Figure 5B). The nutrition metabolism included carbohydrate metabolism, amino 


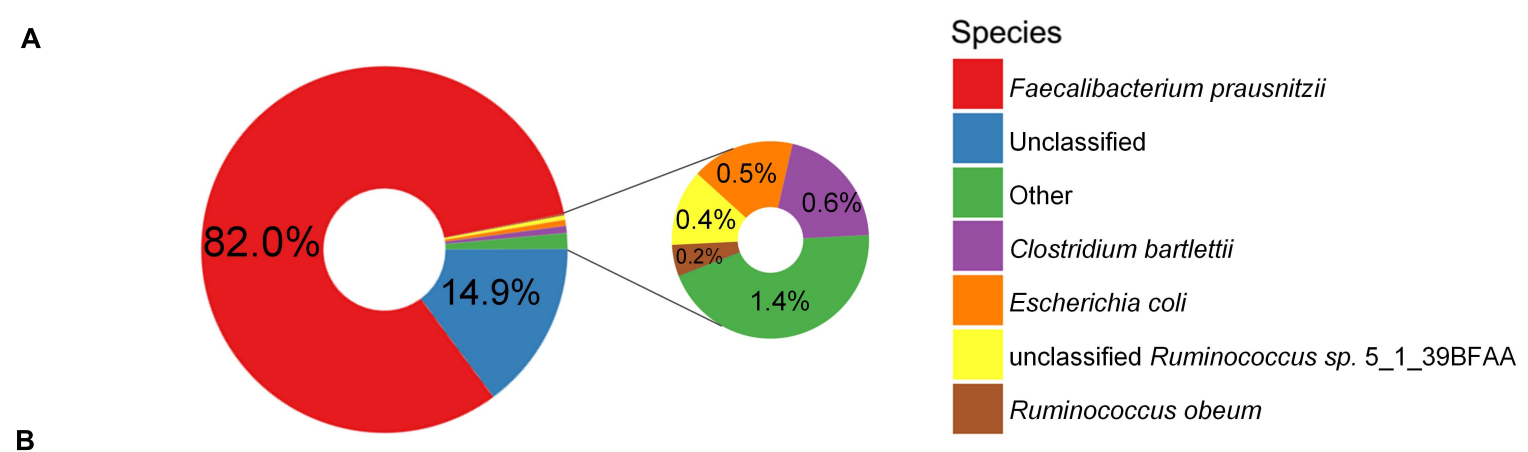

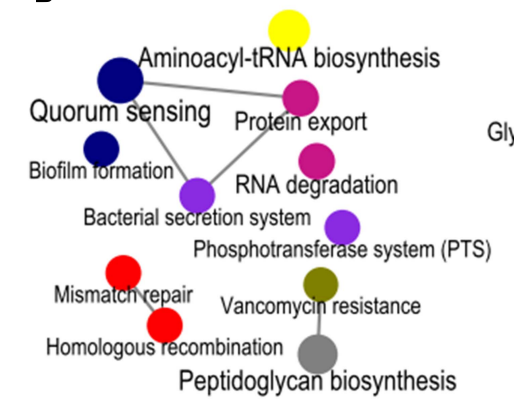

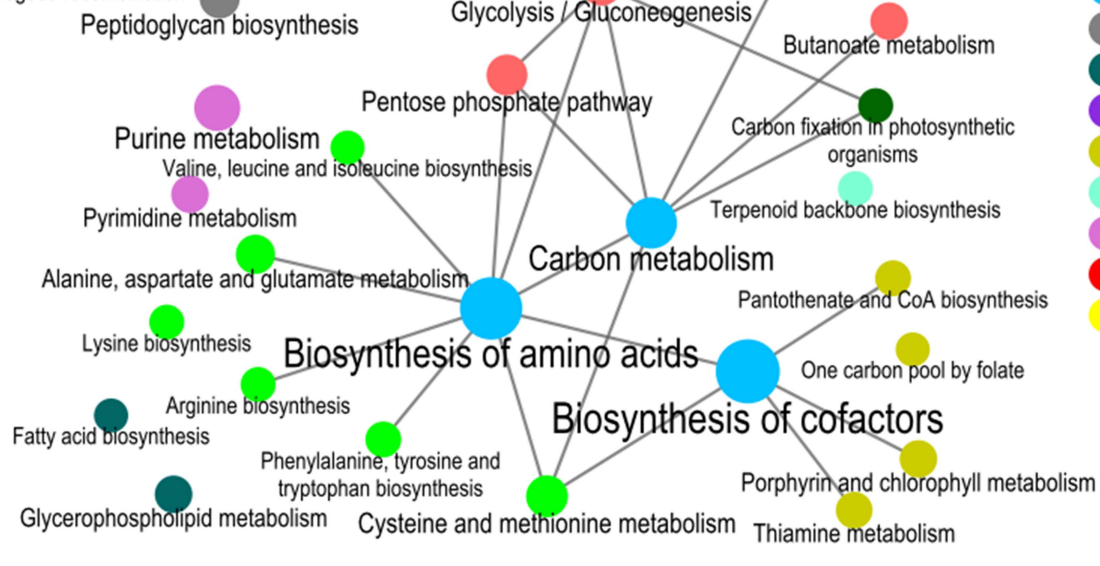

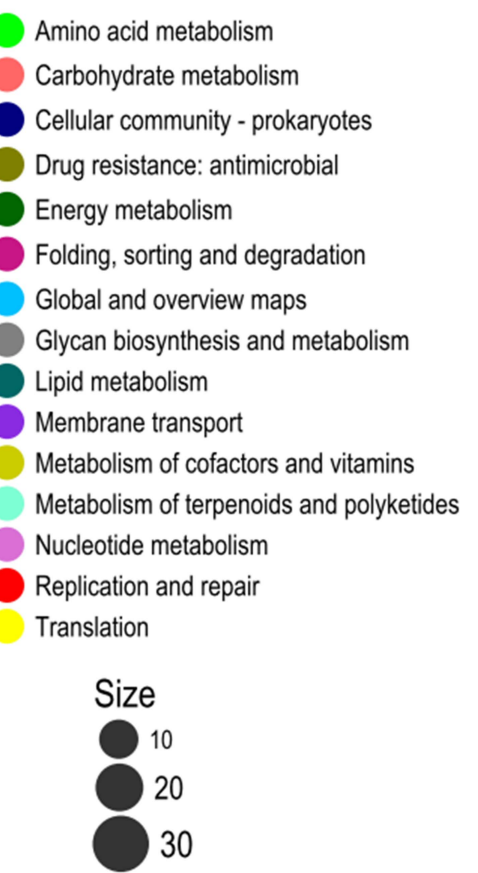

FIGURE 4 | Species composition and enrichment metabolic pathways carrying the BiasSNPs in PWS children. (A) Composition of species with BiasSNP in PWS children. Colors represent different species. (B) The relationship between the metabolic pathways of BiasSNP in PWS children. The line indicates that the metabolic pathways share the same gene, the dot represents the pathway, the size of the dot represents the number of genes with BiasSNP in the pathway, and the color represents the class of pathway.

acid metabolism, metabolism of cofactors and vitamins, and nucleotide metabolism. The SNPs related to DNA replication and repair may furtherly influence the genetic variation, including base excision repair, homologous recombination, mismatch repair, and nucleotide excision repair. In addition, a number of nonsynonymous BiasSNP existed in translation-related metabolic pathways, such as aminoacyl-tRNA biosynthesis and ribosome. Twenty-six genes with nonsynonymous BiasSNPs in the pathway network of SO children are presented in Supplementary Table 2 .

\section{DISCUSSION}

Our previous study performed a dietary intervention trial on obese children with PWS and SO, and found that the high-fiber diet had improved significantly the physiological indexes of all the obese children and changed the composition and structure of the gut microbiota (Zhang et al., 2015). This study focused on the gut microbial SNP variations that occurred in genes, trying to figure out important genes and functional strains influenced by the intervention.

We found that the remarkable changes in gut microbial SNPs caused by the intervention were related to nutrition metabolism, including carbohydrate metabolism (e.g., gluconeogenesis and pyruvate metabolism), amino acid metabolism, and lipid metabolism in all obese children. This result was not surprising because the SNPs existed densely in strains to adapt to environmental changes. Compared with the normal diet, the WTP diet provides large quantities of whole-grain mix that is rich in starch, soluble and insoluble dietary fiber, protein, and amino acids, but contains a small amount of fat (Xiao et al., 2014). When 
A

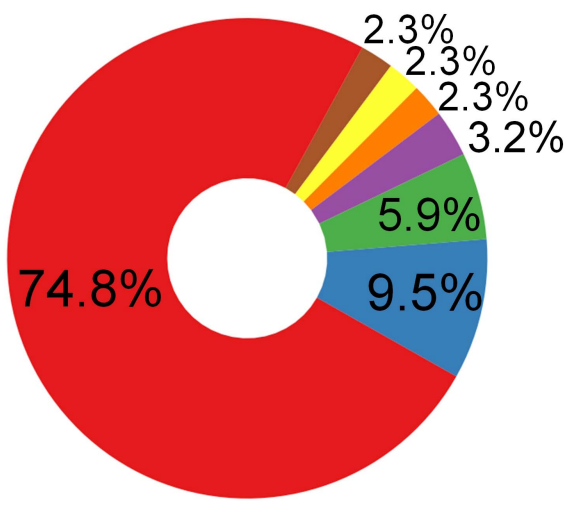

Species

Faecalibacterium prausnitzii

Unclassified

Other

Bifidobacterium longum

Bifidobacterium adolescentis

Clostridium difficile

Streptococcus thermophilus
B

Mismatch repair Homologous recombination

Nucleotide excision repair

Phosphotransferase system (PTS)

RNA polymerase

Autophagy

Biosynthesis of cofactors Purine metabolism

Aminoacyl-tRNA biosynthesis

Ribosome

Pyrimidine metabolism

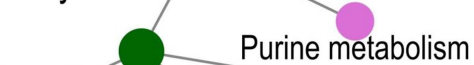

Sulfur relay system

Thiamine metabolism

Biofilm formation Riboflavin metabolism

Starch and sucrose metabolism

Pantothenate and CoA biosynthesis

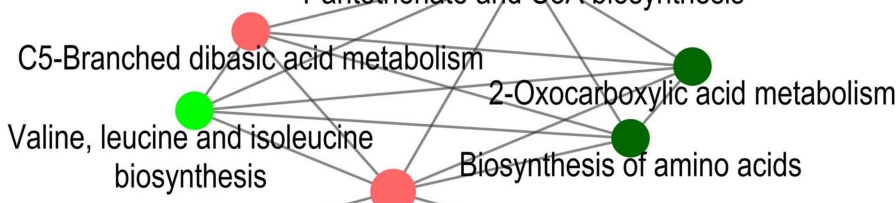

Butanoate metabolism

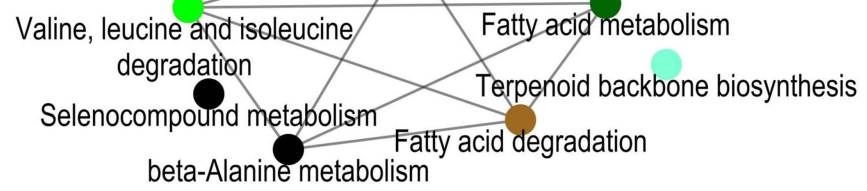

Amino acid metabolism

Carbohydrate metabolism

Cellular community - prokaryotes

Transport and catabolism

- Transcription

Folding, sorting and degradation

Global and overview maps

Metabolism of other amino acids

Lipid metabolism

Membrane transport

Metabolism of cofactors and vitamins

Metabolism of terpenoids and polyketides

Nucleotide metabolism

Replication and repair

Translation

\section{Size}

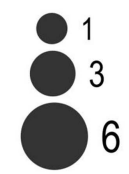

FIGURE 5 | Species composition and enrichment metabolic pathways carrying the BiasSNPs in SO children. (A) Composition of species with BiasSNP in PWS children. Colors represent different species. (B) The relationship between the metabolic pathways of BiasSNP in PWS children. The line indicates that the metabolic pathways share the same gene, the dot represents the pathway, the size of the dot represents the number of genes with BiasSNP in the pathway, and the color represents the class of pathway.

this excess and/or indigestible nutrition reached the colon, they brought environmental pressures to the microbiota that stayed there. This pressure could facilitate the utilization of indigestible nutrition by causing microbial SNPs and, thus, affecting the functions of the related genes (such as $p y k$, coding the pyruvate kinase). As a result, the metabolic efficiencies of indigestible nutrition substrates would be enhanced to adapt to the shifted environment better. Conversely, as the WTP diet is low in fat, the lower lipid substrate level in the intestinal environment might lead to SNPs in lipid metabolism-relevant genes (such as fadD, coding long-chain acyl-CoA synthetase) and, thus, would furtherly reduce the efficiencies of lipid nutrition substrates.
Meanwhile, SNPs also emerged in some pathways related to the adaptability to environmental changes and the virulence of bacteria, such as the two-component system, transport system, secretion system, and drug tolerance. The two-component system is a signal transduction system widely existing in bacteria, which plays an important role in responding to the constantly changing environment by means of protein phosphorylation (Wang et al., 2002; Zuniga et al., 2011). ABC transport system utilizes the energy released by ATP hydrolysis to transport various substrates across membranes, including amino acid, sugar, lipid, polypeptide, alcohol, metal, drug, etc. (Koster, 2001; Hollenstein et al., 2007). Additionally, the ABC transport system 
is also involved in some other biological processes, such as RNA translation and DNA repair (Licht and Schneider, 2011). HlyD, a member of the membrane fusion proteins (MFP), links the inner and outer membranes in some way by spanning the periplasm and is necessary for the secretion of repeats in toxin (RTX) hemolytic toxins (Pimenta et al., 1999; Pimenta et al., 2005). RTX is a kind of high-molecular weight protein, heatresistant, calcium-dependent toxin, secreted by a large class of Gram-negative pathogens, which can lysis various creatural target cells (Lally et al., 1999). Dietary changes brought drastic variations to the intestinal environment and intense evolutionary pressure on the gut microbiota in obese children. Though further validation is needed, our results implied that, in response to this environmental pressure, some gut microbial SNPs that occurred might affect the efficiency and function of metabolic pathways related to environmental adaptation, and might be relevant to the health promotion of the obese children.

Previous studies had observed that Bifidobacterium was the selectively promoted genera under the WTP intervention due to their outperforming ability to utilize carbohydrates (Xiao et al., 2014; Zhang et al., 2015; Zhao et al., 2018). Indeed, Bifidobacterium became dominant strains responsible for nutrition metabolism after the intervention in both PWS and SO children based on SNP density analysis. Bifidobacterium has been demonstrated to have various probiotic effects on the health of the host, involving protection of the intestinal barrier, modulation of the immune response, and effects of antimicrobial, anti-inflammatory, and anti-obesity (Marteau et al., 2001; Heuvelin et al., 2009; Turroni et al., 2014). However, when we turned to BiasSNP analysis, unexpectedly, it was Faecalibacterium, instead of Bifidobacterium, that had the most nonsynonymous SNPs, suggesting that the intervention mainly affected the functional mutations of Faecalibacterium, especially $F$. prausnitzii. F. prausnitzii can reduce the synthesis of colonic pro-inflammatory cytokines, induce the secretion of anti-inflammatory cytokines, and inhibit the activation of NF$\kappa \mathrm{B}$ and the production of IL-8 (Sokol et al., 2008; Miquel et al., 2015), and produce butyrate to make protective and antiinflammatory effects (Ohira et al., 2017). In addition, the track to strains carrying BiasSNPs showed that the dominant strains, F. prausnitzii AF32-8AC and F. prausnitzii 942/30-2, converted to $F$. prausnitzii SSTS Bg7063 and F. prausnitzii JG BgPS064 after the dietary intervention, indicating that dietary intervention probably changed the dominant strains of F. prausnitzii by changing the intestinal environment. If the study only focuses on the composition or abundance of the gut microbiota as the mainstream used, the important information about $F$. prausnitzii would be neglected. A previous study of $31 \mathrm{~F}$. prausnitzii genomes reported that the functional differences among these strains were mainly concentrated in the metabolism of carbohydrates and amino acids (Fitzgerald et al., 2018). However, we found that the functional differences of $F$. prausnitzii were not only on nutrition metabolism but also in response to environmental changes, such as signal transduction and membrane transport. This additional finding suggested that SNP analysis on gut microbiota could provide more details about the functions and characteristics at the strain level.
Some differences in the gut microbial SNP existed between PWS and SO children in response to the intervention. There were more BiasSNPs between before and after intervention in PWS children than in SO, suggesting that the influence of high-fiber diet on the gut microbial SNP may be greater in PWS than in SO. PWS-specific BiasSNPs were mainly related to nutrition metabolism, protein transport, and environmental adaptation. The phosphotransferase system (PTS) in bacteria can transport carbohydrates into cells by phosphorylation (Deutscher et al., 2007), and also perceive available carbohydrates and intracellular energy, regulate the decomposition of metabolites, and ensure the optimal utilization efficiency of carbohydrates in a complex environment (Lengeler, 1996; Kotrba et al., 2001). N-acetylgalactosamine-specific PTS, which correlated with the nonsynonymous BiasSNPs in PWS, is a common amino-sugar transport system in the gut microbiota, which can regulate and transport acetyl galactosamine (Brinkkotter et al., 2000; Ezquerro-Saenz et al., 2006). Additionally, PWSspecific BiasSNPs were also concentrated in two kinds of proteins translocation systems, general secretory (Sec) pathway and twin-arginine translocation (Tat) system. The Sec pathway is a common and universal protein translocation system, which could integrate synthetic proteins into bacterial cell membranes (Zhou et al., 2014; Tsirigotaki et al., 2017), while the Tat system can transport folded proteins efficiently across the cytoplasmic membranes (Palmer et al., 2010). Moreover, PWSspecific BiasSNPs were also related to spore formation, which is wrapped by a layer of complex macromolecular protein shell under special conditions to resist the hydrolysis of enzyme and protect the active molecules (Setlow, 2003; Kim et al., 2006). These differences in gut microbial SNPs between PWS and SO could not be discovered if only composition information was used, which emphasized the importance of SNP analysis again.

In this work, the identified non-synonymous SNPs were dominantly carried by $F$. prausnitzii strains. Though F. prausnitzii were well known for their biodiversity, we could not find functional reports about these SNPs. Future efforts are needed to verify/discern the specific effects of these SNPs on the encoded protein activity, their role on metabolism under high-fiber dietary intervention, and their potential beneficial or detrimental influences on host health. The verification/discernment could be done through combining molecular simulation or experimental design.

\section{CONCLUSION}

Our results demonstrated that the high-fiber dietary intervention altered the gut microbial SNP patterns in obese children, and intervened the efficiency and function of metabolic pathways in nutrition metabolism and environmental adaptation. F. prausnitzii had been screened out as the dominant strains by changing multiple functional SNPs under the intervention, which had the potential to improve obesity and could be used as a probiotic supplementary in the prevention and treatment of obesity. This bioinformatics study provided evidence for the influence of dietary intervention on gut microbial SNPs, 
highlighted the importance of SNP analysis on searching differential genes and functional strains from complexed microbial ecosystem, and gave some enlightenment for obesity or other disease treatment.

\section{DATA AVAILABILITY STATEMENT}

The original contributions presented in the study are included in the article/Supplementary Material, further inquiries can be directed to the corresponding author/s.

\section{ETHICS STATEMENT}

The studies involving human participants were reviewed and approved by the Chinese Clinical Trial Registry. Written informed consent to participate in this study was provided by the participants' legal guardian/next of kin.

\section{REFERENCES}

Baothman, O. A., Zamzami, M. A., Taher, I., Abubaker, J., and Abu-Farha, M. (2016). The role of gut microbiota in the development of obesity and diabetes. Lipids Health Dis. 15:108. doi: 10.1186/s12944-016-0278-4

Brinkkotter, A., Kloss, H., Alpert, C., and Lengeler, J. W. (2000). Pathways for the utilization of N-acetyl-galactosamine and galactosamine in Escherichia coli. Mol. Microbiol. 37, 125-135. doi: 10.1046/j.1365-2958.2000.01969.x

Chen, Y. W., Li, Z. C., Hu, S. F., Zhang, J., Wu, J. Q., Shao, N. S., et al. (2017). Gut metagenomes of type 2 diabetic patients have characteristic single-nucleotide polymorphism distribution in Bacteroides coprocola. Microbiome 5:15. doi: 10. 1186/s40168-017-0232-3

Deutscher, J., Francke, C., and Postma, P. W. (2007). How phosphotransferase system-related protein phosphorylation regulates carbohydrate metabolism in bacteria. Microbiol. Mol. Biol. Rev. 70, 939-1031. doi: 10.1128/Mmbr.00024-06

Dodt, M., Roehr, J. T., Ahmed, R., and Dieterich, C. (2012). FLEXBAR-Flexible barcode and adapter processing for next-generation sequencing platforms. Biology (Basel) 1, 895-905. doi: 10.3390/biology1030895

Ezquerro-Saenz, C., Ferrero, M. A., Revilla-Nuin, B., Lopez Velasco, F. F., Martinez-Blanco, H., and Rodriguez-Aparicio, L. B. (2006). Transport of $\mathrm{N}$-acetyl-D-galactosamine in Escherichia coli K92: effect on acetyl-amino sugar metabolism and polysialic acid production. Biochimie 88, 95-102. doi: 10.1016/ j.biochi.2005.06.011

Fitzgerald, C. B., Shkoporov, A. N., Sutton, T. D. S., Chaplin, A. V., Velayudhan, V., Ross, R. P., et al. (2018). Comparative analysis of Faecalibacterium prausnitzii genomes shows a high level of genome plasticity and warrants separation into new species-level taxa. BMC Genomics 19:931. doi: 10.1186/s12864-018-5313-6

Flint, H. J. (2011). Obesity and the gut microbiota. J. Clin. Gastroenterol. 45 Suppl, S128-S132. doi: 10.1097/MCG.0b013e31821f44c4

Galloway-Peña, J., Roh, J. H., Latorre, M., Qin, X., and Murray, B. E. (2012). Genomic and SNP analyses demonstrate a distant separation of the hospital and community-associated clades of Enterococcus faecium. PLoS One 7:e30187. doi: 10.1371/journal.pone.0030187

Goldsmith, J. R., and Sartor, R. B. (2014). The role of diet on intestinal microbiota metabolism: downstream impacts on host immune function and health, and therapeutic implications. J. Gastroenterol. 49, 785-798. doi: 10.1007/s00535014-0953-Z

Gubler, M., Jetten, M., Lee, S. H., and Sinskey, A. J. (1994). Cloning of the pyruvate kinase gene (pyk) of Corynebacterium glutamicum and site-specific inactivation of pyk in a lysine-producing Corynebacterium lactofermentum strain. Appl. Environ. Microbiol. 60, 2494-2500. doi: 10.1128/aem.60.7.2494-2500.1994

\section{AUTHOR CONTRIBUTIONS}

MZ, LZ, and HL contributed to conception and design of the study. HL performed the statistical analysis and wrote the first draft of the manuscript. All authors contributed to the manuscript revision and approved the submitted version.

\section{ACKNOWLEDGMENTS}

We would like to thank the members of the MOLECO Lab at Shanghai Jiao Tong University for comments and suggestions on the analysis.

\section{SUPPLEMENTARY MATERIAL}

The Supplementary Material for this article can be found online at: https://www.frontiersin.org/articles/10.3389/fmicb. 2021.683714/full\#supplementary-material

Haraksingh, R. R., and Snyder, M. P. (2013). Impacts of variation in the human genome on gene regulation. J. Mol. Biol. 425, 3970-3977. doi: 10.1016/j.jmb. 2013.07.015

Heuvelin, E., Lebreton, C., Grangette, C., Pot, B., Cerf-Bensussan, N., and Heyman, M. (2009). Mechanisms involved in alleviation of intestinal inflammation by Bifidobacterium breve soluble factors. PLoS One 4:e5184. doi: 10.1371/journal. pone.0005184

Hofreiter, M., Paijmans, J. L., Goodchild, H., Speller, C. F., Barlow, A., Fortes, G. G., et al. (2015). The future of ancient DNA: technical advances and conceptual shifts. Bioessays 37, 284-293. doi: 10.1002/bies.201400160

Hollenstein, K., Dawson, R. J., and Locher, K. P. (2007). Structure and mechanism of ABC transporter proteins. Curr. Opin. Struct. Biol. 17, 412-418. doi: 10.1016/ j.sbi.2007.07.003

Hu, Y., Feng, Y., Wu, J., Liu, F., Zhang, Z., Hao, Y., et al. (2019). The gut microbiome signatures discriminate healthy from pulmonary tuberculosis patients. Front. Cell Infect. Microbiol. 9:90. doi: 10.3389/fcimb.2019.00090

Hu, Y., Yang, X., Qin, J., Lu, N., Cheng, G., Wu, N., et al. (2013). Metagenome-wide analysis of antibiotic resistance genes in a large cohort of human gut microbiota. Nat. Commun. 4:2151. doi: 10.1038/ncomms3151

Kim, H., Hahn, M., Grabowski, P., McPherson, D. C., Otte, M. M., Wang, R., et al. (2006). The Bacillus subtilis spore coat protein interaction network. Mol. Microbiol. 59, 487-502. doi: 10.1111/j.1365-2958.2005.04968.x

Koster, W. (2001). ABC transporter-mediated uptake of iron, siderophores, heme and vitamin B12. Res. Microbiol. 152, 291-301. doi: 10.1016/s0923-2508(01) 01200-1

Kotrba, P., Inui, M., and Yukawa, H. (2001). Bacterial phosphotransferase system (PTS) in carbohydrate uptake and control of carbon metabolism. J. Biosci. Bioeng. 92, 502-517. doi: 10.1263/jbb.92.502

Lally, E. T., Hill, R. B., Kieba, I. R., and Korostoff, J. (1999). The interaction between RTX toxins and target cells. Trends Microbiol. 7, 356-361. doi: 10.1016/s0966$842 \times(99) 01530-9$

Langmead, B., and Salzberg, S. L. (2012). Fast gapped-read alignment with Bowtie 2. Nat. Methods 9, 357-359. doi: 10.1038/nmeth.1923

Lengeler, J. W. (1996). “The Phosphoenolpyruvate-Dependent Carbohydrate: Phosphotransferase System (PTS) and Control of Carbon Source Utilization," in Regulation of Gene Expression in Escherichia coli, eds E. C. C. Lin, and A. Simon Lynch (Boston, MA: Springer US), 231-254.

Leonard, S. R., Mammel, M. K., Lacher, D. W., and Elkins, C. A. (2016). Strainlevel discrimination of Shiga toxin-producing Escherichia coli in spinach using metagenomic sequencing. PLoS One 11:e0167870. doi: 10.1371/journal.pone. 0167870 
Li, H. (2011). A statistical framework for SNP calling, mutation discovery, association mapping and population genetical parameter estimation from sequencing data. Bioinformatics 27, 2987-2993. doi: 10.1093/bioinformatics/ btr509

Li, H., and Durbin, R. (2009). Fast and accurate short read alignment with BurrowsWheeler transform. Bioinformatics 25, 1754-1760. doi: 10.1093/bioinformatics/ btp324

Li, H., Handsaker, B., Wysoker, A., Fennell, T., Ruan, J., Homer, N., et al. (2009). The sequence alignment/map format and SAMtools. Bioinformatics 25, 20782079. doi: 10.1093/bioinformatics/btp352

Li, J., Jia, H., Cai, X., Zhong, H., Feng, Q., Sunagawa, S., et al. (2014). An integrated catalog of reference genes in the human gut microbiome. Nat. Biotechnol. 32, 834-841. doi: 10.1038/nbt.2942

Licht, A., and Schneider, E. (2011). ATP binding cassette systems: structures, mechanisms, and functions. Open Life Sci. 6, 785-801.

Marteau, P. R., de Vrese, M., Cellier, C. J., and Schrezenmeir, J. (2001). Protection from gastrointestinal diseases with the use of probiotics. Am. J. Clin. Nutr. 73(2 Suppl), 430S-436S. doi: 10.1093/ajcn/73.2.430s

Miquel, S., Leclerc, M., Martin, R., Chain, F., Lenoir, M., Raguideau, S., et al. (2015). Identification of metabolic signatures linked to anti-inflammatory effects of Faecalibacterium prausnitzii. mBio 6:e00300-15. doi: 10.1128/mBio.00300-15

Nayfach, S., Rodriguez-Mueller, B., Garud, N., and Pollard, K. S. (2016). An integrated metagenomics pipeline for strain profiling reveals novel patterns of bacterial transmission and biogeography. Genome Res. 26, 1612-1625. doi: 10.1101/gr.201863.115

Ohira, H., Tsutsui, W., and Fujioka, Y. (2017). Are short chain fatty acids in gut microbiota defensive players for inflammation and atherosclerosis? J. Atheroscler. Thromb. 24, 660-672. doi: 10.5551/jat.RV17006

Palmer, T., Sargent, F., and Berks, B. C. (2010). The Tat protein export pathway. EcoSal Plus 4, 1-35. doi: 10.1128/ecosalplus.4.3.2

Pech-Canul, A. C., Rivera-Hernandez, G., Nogales, J., Geiger, O., Soto, M. J., and Lopez-Lara, I. M. (2020). Role of Sinorhizobium meliloti and Escherichia coli long-chain acyl-CoA synthetase FadD in long-term survival. Microorganisms 8:470. doi: 10.3390/microorganisms 8040470

Pimenta, A. L., Racher, K., Jamieson, L., Blight, M. A., and Holland, I. B. (2005). Mutations in HlyD, part of the type 1 translocator for hemolysin secretion, affect the folding of the secreted toxin. J. Bacteriol. 187, 7471-7480. doi: 10.1128/jb. 187.21.7471-7480.2005

Pimenta, A. L., Young, J., Holland, I. B., and Blight, M. A. (1999). Antibody analysis of the localisation, expression and stability of HlyD, the MFP component of the E. coli haemolysin translocator. Mol. Gen. Genet. 261, 122-132. doi: 10.1007/ s004380050949

Schmieder, R., and Edwards, R. (2011). Quality control and preprocessing of metagenomic datasets. Bioinformatics 27, 863-864. doi: 10.1093/ bioinformatics/btr026

Setlow, P. (2003). Spore germination. Curr. Opin. Microbiol. 6, 550-556. doi: 10. 1016/j.mib.2003.10.001

Shannon, P., Markiel, A., Ozier, O., Baliga, N. S., Wang, J. T., Ramage, D., et al. (2003). Cytoscape: a software environment for integrated models of biomolecular interaction networks. Genome Res. 13, 2498-2504. doi: 10.1101/ gr.1239303

Shen, T. D. (2017). Diet and gut microbiota in health and disease. Nestle Nutr. Inst. Workshop Ser. 88, 117-126. doi: 10.1159/000455220

Sokol, H., Pigneur, B., Watterlot, L., Lakhdari, O., Bermudez-Humaran, L. G., Gratadoux, J. J., et al. (2008). Faecalibacterium prausnitzii is an antiinflammatory commensal bacterium identified by gut microbiota analysis of Crohn disease patients. Proc. Natl. Acad. Sci. U.S.A. 105, 16731-16736. doi: 10.1073/pnas.0804812105

Stamatakis, A. (2014). RAxML version 8: a tool for phylogenetic analysis and post-analysis of large phylogenies. Bioinformatics 30, 1312-1313. doi: 10.1093/ bioinformatics/btu033
Tennessen, J. A. (2008). Positive selection drives a correlation between non-synonymous/synonymous divergence and functional divergence. Bioinformatics 24, 1421-1425. doi: 10.1093/bioinformatics/btn205

Truong, D. T., Tett, A., Pasolli, E., Huttenhower, C., and Segata, N. (2017). Microbial strain-level population structure and genetic diversity from metagenomes. Genome Res. 27, 626-638. doi: 10.1101/gr.216242.116

Tsirigotaki, A., De Geyter, J., Sostaric, N., Economou, A., and Karamanou, S. (2017). Protein export through the bacterial Sec pathway. Nat. Rev. Microbiol. 15, 21-36. doi: 10.1038/nrmicro.2016.161

Turnbaugh, P. J., Ley, R. E., Mahowald, M. A., Magrini, V., Mardis, E. R., and Gordon, J. I. (2006). An obesity-associated gut microbiome with increased capacity for energy harvest. Nature 444, 1027-1031. doi: 10.1038/nature0 5414

Turroni, F., Ventura, M., Buttó, L. F., Duranti, S., O’Toole, P. W., Motherway, M. O., et al. (2014). Molecular dialogue between the human gut microbiota and the host: a Lactobacillus and Bifidobacterium perspective. Cell. Mol. Life Sci. 71, 183-203. doi: 10.1007/s00018-013-1318-0

Wang, L., Sun, Y. P., Chen, W. L., Li, J. H., and Zhang, C. C. (2002). Genomic analysis of protein kinases, protein phosphatases and two-component regulatory systems of the cyanobacterium Anabaena sp. strain PCC 7120. FEMS Microbiol. Lett. 217, 155-165. doi: 10.1111/j.1574-6968.2002.tb11469.x

Xiao, S., Fei, N., Pang, X., Shen, J., Wang, L., Zhang, B., et al. (2014). A gut microbiota-targeted dietary intervention for amelioration of chronic inflammation underlying metabolic syndrome. FEMS Microbiol. Ecol. 87, 357367. doi: 10.1111/1574-6941.12228

Xie, H., Guo, R., Zhong, H., Feng, Q., Lan, Z., Qin, B., et al. (2016). Shotgun metagenomics of 250 adult twins reveals genetic and environmental impacts on the gut microbiome. Cell Syst. 3, 572-584.e3. doi: 10.1016/j.cels.2016. 10.004

Yu, G., Wang, L. G., Han, Y., and He, Q. Y. (2012). clusterProfiler: an R package for comparing biological themes among gene clusters. OMICS 16, 284-287. doi: 10.1089/omi.2011.0118

Zhang, C., Yin, A., Li, H., Wang, R., Wu, G., Shen, J., et al. (2015). Dietary modulation of gut microbiota contributes to alleviation of both genetic and simple obesity in children. EBioMedicine 2, 968-984. doi: 10.1016/j.ebiom.2015. 07.007

Zhao, L., Zhang, F., Ding, X., Wu, G., Lam, Y. Y., Wang, X., et al. (2018). Gut bacteria selectively promoted by dietary fibers alleviate type 2 diabetes. Science 359, 1151-1156. doi: 10.1126/science.aao5774

Zhou, Z., Li, Y., Sun, N., Sun, Z., Lv, L., Wang, Y., et al. (2014). Function and evolution of two forms of SecDF homologs in Streptomyces coelicolor. PLoS One 9:e105237. doi: 10.1371/journal.pone.0105237

Zou, D., Pei, J., Lan, J., Sang, H., Chen, H., Yuan, H., et al. (2020). A SNP of bacterial blc disturbs gut lysophospholipid homeostasis and induces inflammation through epithelial barrier disruption. EBioMedicine 52:102652. doi: 10.1016/j. ebiom.2020.102652

Zuniga, M., Gomez-Escoin, C. L., and Gonzalez-Candelas, F. (2011). Evolutionary history of the OmpR/IIIA family of signal transduction two component systems in Lactobacillaceae and Leuconostocaceae. BMC Evol. Biol. 11:34. doi: 10.1186/ 1471-2148-11-34

Conflict of Interest: The authors declare that the research was conducted in the absence of any commercial or financial relationships that could be construed as a potential conflict of interest.

Copyright (c) $2021 \mathrm{Li}$, Zhao and Zhang. This is an open-access article distributed under the terms of the Creative Commons Attribution License (CC BY). The use, distribution or reproduction in other forums is permitted, provided the original author(s) and the copyright owner(s) are credited and that the original publication in this journal is cited, in accordance with accepted academic practice. No use, distribution or reproduction is permitted which does not comply with these terms. 